\title{
CONFERENCE SUMMARY: THE LEGACY OF AKARI: A PANORAMIC VIEW OF THE DUSTY UNIVERSE
}

\author{
GLenn J. White ${ }^{1,2}$ \\ ${ }^{1}$ Department of Physical Sciences, The Open University, Milton Keynes MK7 6AA, England, \\ ${ }^{2}$ RALSpace, The Rutherford Appleton Laboratory, Chilton, Didcot, Oxfordshire OX11 0NL, England \\ E-mail: g.j.white@open.ac.uk \\ (Received July 08, 2012; Accepted August 15, 2012)
}

\begin{abstract}
This report presents a summary of the Legacy of AKARI: A Panoramic View of the Dusty Universe meeting held between 27-29th February 2012 at Jeju Island, South Korea.
\end{abstract}

Key words: infrared: telescope; conferences: proceedings

\section{INTRODUCTION}

AKARI was launched on February 21, 2006 with the objective of carrying out all sky surveys at mid- and far-infrared wavelengths between 9 and $160 \mu \mathrm{m}$, and pointed observations to obtain ultra-deep surveys of the distant Universe across this broad wavelength interval. Operating for approximately 550 days at cryogenic temperatures until August 26, 2007, it then continued as a warm near-IR mission until recently.

The first dedicated conference presenting results from the AKARI mission was held between 16-19 February, 2009, at the University of Tokyo, Japan. Since then, almost 600 papers based on AKARI results have been published in the literature, including in special issues of The Publications of The Astronomical Society of Japan, Astronomy and Astrophysics, and other major international journals. This Conference has once again brought together researchers working on the data obtained by AKARI to discuss the impact of its major new scientific results in areas such as the interstellar medium, evolved stars, nearby galaxies and the distant Universe, and to plan future research directions following from this highly successful mission.

Scientific areas covered during the Conference included:

- The Survey Legacy of AKARI
- The Solar System

- Stars and Circumstellar Matter

- The Interstellar Medium and Interstellar dust

- Nearby galaxies

- Distant galaxies

- Synergies and the future

This scientific meeting has brought together a large international consortium which has a) contributed to burgeoning communities in Korea and Japan, b) engaged with major international space agencies, such as JAXA, ESA and the UK's STFC, c) spawned may scientific collaborations and friendships over more than a decade, d) achieved some really unique scientific breakthroughs, not previously available though any other facilities of missions, e) has been a credit to the vision, drive, enthusiasm and creativity of many of the team members, especially in Japan, whose hard work has led to the success and the Legacy of AKARI. The meeting was attended by 55 Japanese, 29 Korean, 7 British, 5 American, 4 Polish, 3 French and 1 Finnish astronomers, showing the true spirit of international participation and collaboration that was fostered, both during and after the AKARI mission, and also at this timely conference on Jeju Island, Korea (Figure 1). 


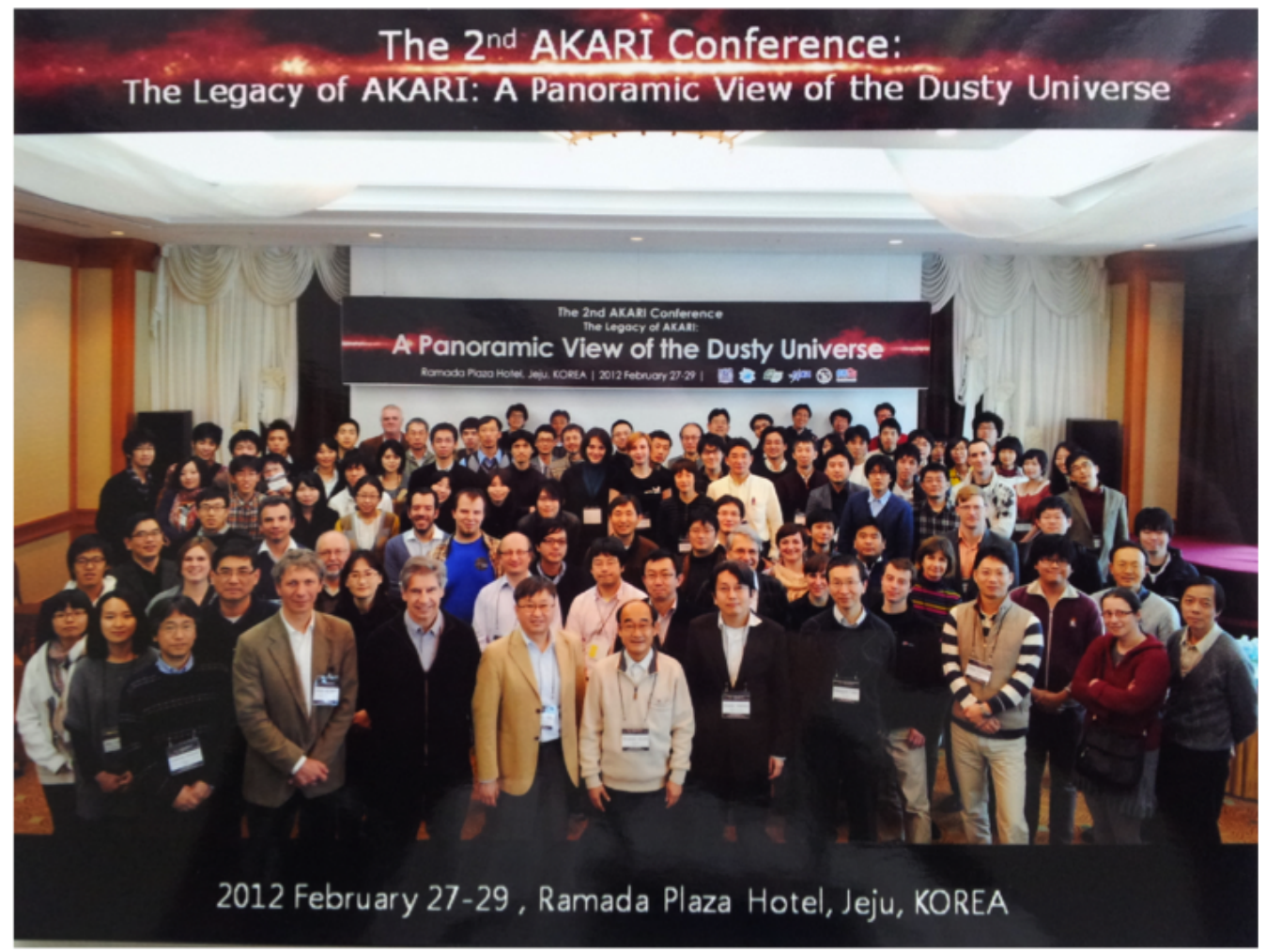

Fig. 1. Conference participants at the 2nd AKARI Conference.

\section{THE SURVEY LEGACY OF AKARI}

An unique aspect of AKARI's Legacy has been the release of its All Sky Survey catalogues for the international community (Figure 2), delivering the first All Sky far-IR survey since ESA's Infrared Astronomy Satellite (IRAS) in the 1980's. At this conference (papers from all of the authors in this summary will be found in this special issue of The Publications of The Korean Astronomical Society), Ishihara et al. presented the AKARI MIR All-Sky Point Source Catalogue, at wavelengths of 9 and $18 \mu$ m containing 870,000 sources, showing convincing evidence that the distributions of C- and O-rich AGB stars differ, and suggesting that they were related to the metallicity of their birthplaces. At longer wavelengths Yamamura et al. presented the bright source catalogues detected in the Far Infrared Surveyor (FIS) All Sky Survey at 65, 90, 140 and $160 \mu \mathrm{m}$, containing $\sim 430,000$ sources. To these already published catalogues, Doi et al. reported a new Survey product that is being prepared for release during 2012, of an All-Sky Diffuse map. An early version of the $90 \mu \mathrm{m}$ map from this new data product is shown in Figure 3.

\section{THE SOLAR SYSTEM}

Usui et al. presented spectacular movie simulations that provided a panoramic view of bodies in the inner solar system viewed by AKARI, and argued that asteroids are not thermally differentiated, but contain a pristine record of the Solar Systems formation. Pyo et al. presented AKARI observations of the zodiacal cloud, showing additional features such as comets, while Ootsubo et al. reported a near-infrared spectroscopic survey of water and carbon dioxide in comets, postulating that the $\mathrm{CO}_{2} / \mathrm{H}_{2} \mathrm{O}$ production rate ratios trace the composition of cometary ice when a comet was observed at heliocentric distances of $2.5 \mathrm{AU}$.

\section{STARS AND CIRCUMSTELLAR MATTER}

Amongst highlights from the MIR survey, Ishihara et al. showed evidence that the distributions of $\mathrm{C}$ - and O-rich AGB stars differ - suggesting that they were affected by the metallicity of their birthplaces. Izumiura et al. presented the results from a volume limited survey of stars within 300 parsecs, showing beautiful images of dust shells around cool evolved stars. The talk by Ishihara et al. discussed the lifecycle of interstellar dust 


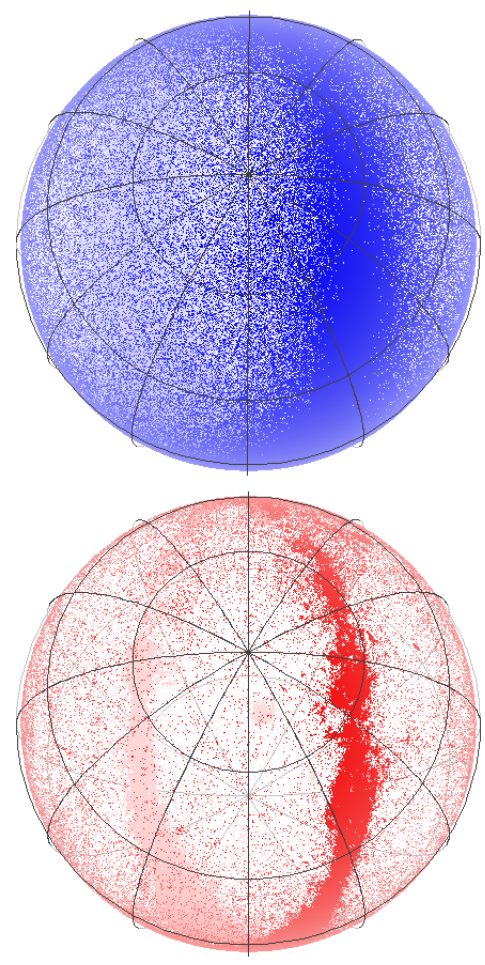

Fig. 2. All sky images at $9 \mu \mathrm{m}$ (blue), and $90 \mu \mathrm{m}$ (red), centred with the north celestial pole just above the centre of the images.

grains in the Galaxy, including a survey of C-rich and O-rich AGB stars - although the C-rich stars are distributed relatively uniformly across the Galactic disk, the O-rich stars show a strong concentration toward the Galactic Centre region. This theme was continued by Szezrba et al. who presented a cross correlation of post-AGB stars with the Torun catalogue. Moving slightly further away, Ita et al. showed results from the new IRC survey of 10 square degrees in the the Large Magellanic Cloud (LMC).

\section{THE INTERSTELLAR MEDIUM AND INTER- STELLAR DUST}

Using a preliminary version of the new All Sky FIS Diffuse survey, Doi et al. and White et al. showed the sensitivity and stability of the diffuse all sky survey in revealing the large scale high Galactic latitude filamentary structures and Gould Belt (Ward-Thompson et al., 2007), whilst Giard et al. explored whether the anomalous microwave emission may be a consequence of rotating Polycyclic Aromatic Hydrocarbon (PAH) grains. AKARI's spectroscopic capability was demon- strated by Shimonishi et al. who reported observations of ices around YSOs in the LMC - showing that the total column density of ices decreases with decreasing metallicity. Fraser et al. showed an unique AKARI blue wing that traced solid state $\mathrm{CO}$ in two different environments - revealing both $\mathrm{CO}$ rich and $\mathrm{H}_{2} \mathrm{O}$ ice layers, particularly at $A_{\mathrm{v}}$ values above 3 magnitudes. Mori et al. showed slitless spectra of unidentified (PAH) bands in the LMC - demonstrating that the ratios of the various UIR lines can serve as a tracer of the interstellar radiation field. and of ionised PAHs in PDRs. Sakon et al. reported spectroscopic observations of diffuse Galactic emission with the IRC instrument showing that the $6.6 / 11.2$ and $7.7 / 11.2 \mu \mathrm{m}$ band ratios in the inner Galactic plane are systematically larger than those in the outer Galaxy.

Koo et al. presented observations of dust modification near SNRs which they associated with the creation fresh formed stardust, crystalline silicates and $\mathrm{FeO}$. Lee et al. showed observations tracing the interaction of the supernova remnant Kes 17 and its nearby molecular clouds - finding signatures of both hot and cold gas, and tracing the interaction physics. Seok et al. reported data tracing 23 supernova remnants in the LMC which had associated IR emission - and which showed a tight IR/X-ray correlation, accompanied by the detection of PAH features - related to the 15-200 $\mu \mathrm{m}$ bump. Onaka et al. continued their impressive work on the ISM showing multi-faceted observations of the ISM, while Kaneda et al. discussed new perspectives on the processing of dust grains on galaxies, postulating that the unusual band ratios observed may be a consequence of the dominance of neutral PAH molecules originating from fragmentation of old grains when close to the centres of their host galaxies.

\section{NEARBY GALAXIES}

Matsuhara et al. reported the identification of a new class of dusty obscured galaxies from the North Ecliptic Pole survey (DOGs), the latter of which is shown in Figure 4, and the subject of many ancillary follow-up studies, e.g. Negrello et al. (2009), White et al. (2010).

Shirahata et al. showed new results on the evolution of IR bright galaxies tracing hot molecular gas with large column densities and systematic molecular outflows, which Nakagawa et al. showed were also associated with X-ray heating, as well as CO absorption features close to $4.6 \mu \mathrm{m}$ in galaxies with obscured AGN 


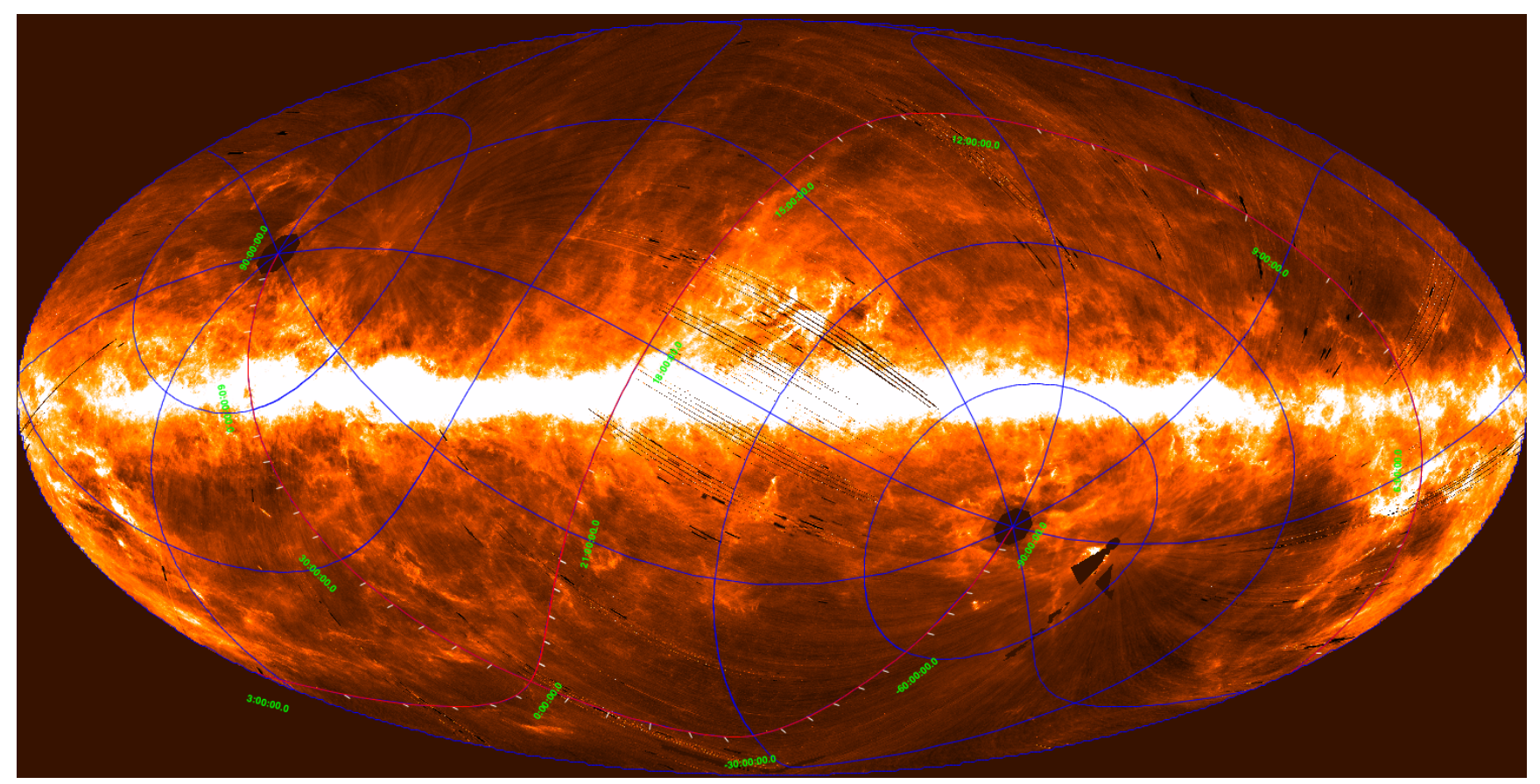

Fig. 3. Diffuse All Sky image in the WIDE-S filter $(90 \mu \mathrm{m})$. The Galactic Plane runs across the centre of this image, and has been saturated so as to show the faint filamentary structure at high Galactic latitudes.

present. Imanishi et al. convincingly showed that luminous buried AGNs are common $(>30 \%)$ in luminous infrared galaxies, and that feedback appeared to have been stronger in the past, effectively quenching star formation. Ichikawa et al. reported observations of a complete sample of local AGN's, concluding the farIR/X-ray correlation strongly supports dusty tori models. They also reported a new class of objects showing excesses close to $9 \mu \mathrm{m}$, which they believed to represent heavily obscured AGNs with small scattering functions in their X-ray spectra. Suzuki et al. presented observations of star formation induced by galaxy-galaxy and galaxy - IGM interactions, showing evidence for gas stripping, leading to an enhancement of the IGM, and evidence for infall. Yamagishi et al. showed AKARI near-IR spectra of interstellar ices in galaxies, which demonstrated that the $\mathrm{CO}_{2}$ ice is often more localised on the galactic nuclear regions that the $\mathrm{H}_{2} \mathrm{O}$ ice. Continuing with nearby galaxies, Egusa et al. showed IRC images of the interacting galaxies in M51, and from ratios in the various filters were able to differentiate warm and cooler dust emitting regions.

\section{DISTANT GALAXIES}

Because of AKARI's polar orbit, two regions of the sky at the North (NEP) and South (SEP) Ecliptic poles, were observed during every orbit, and were used to provide ultra-deep surveys of the distant Universe, which were followed up with extensive observations at other wavelengths to provide complete ancillary data sets. The NEP deep and wide surveys have been a key part of AKARI's key legacy products. Lee et al., Matsuhara et al., and Oyabu et al. reported a survey of dusty AGN's, detecting many showing evidence for hot dust with a colour temperature in excess of $600 \mathrm{~K}$, which likely is a result of central AGN activity. Matsuhara et al. showed detailed analysis of the AKARI and ancillary data sets for the deep North Ecliptic Pole survey, while Matsuura et al. and Clements et al. showed equivalent data sets for the South Ecliptic Pole AKARI Deep Field South (ADF-S) survey. Malek et al. presented results of galaxy SED fitting in the ADF-S, covering 12 square degrees, where more than 9,000 sources were detected at $90 \mu \mathrm{m}$. Pearson et al. showed the importance of using the diffuse All Sky maps in interpreting total galaxy fluxes, and the role of radiative feedback in suppressing star formation which was a common theme that many people referred to. Helou et al. presented 


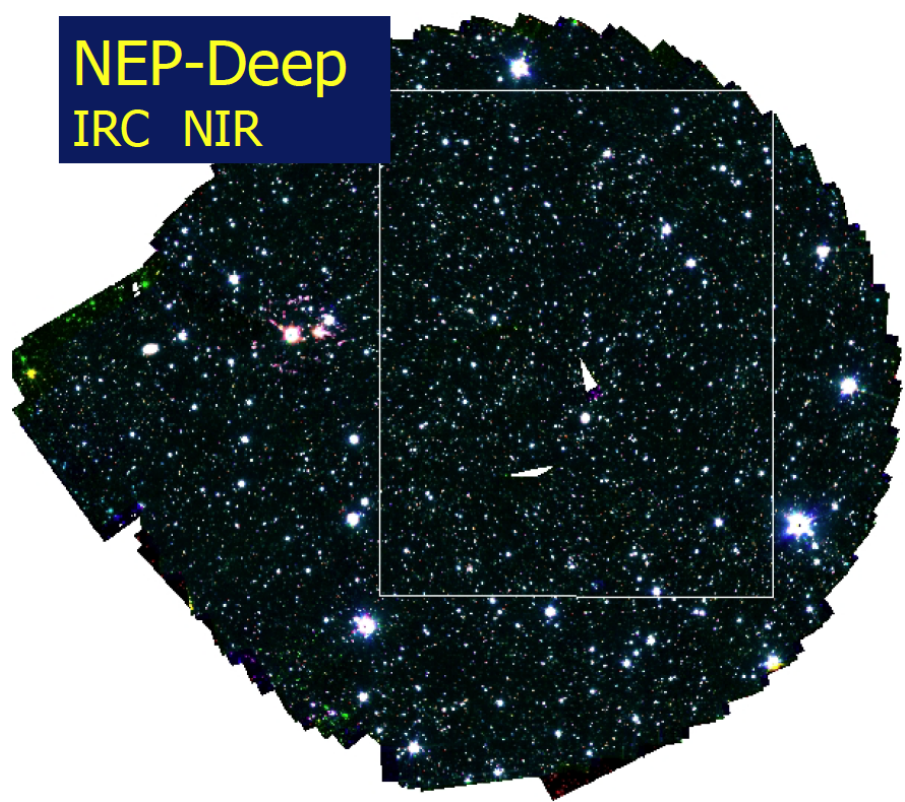

Fig. 4. A three-colour image of NEP-Deep field (Wada et al., 2008), generated from three images taken from the NIR bands of the IRC.

an innovative phenomenological model for black hole growth and star formation, breaking some of the current degeneracies and exploring the relative importance of star formation and accretion onto black holes. Burgarella et al. presented results on Lyman break galaxies in the redshift range $0.7<\mathrm{z}<4$, emphasising their importance in the framework of wider galaxy evolution models, and using the galaxy SED fitting using the Code Investigating GAlaxy Emission (CIGALE) code for the redshift range 0.7-4.2. This demonstrated the uniqueness that the future SPICA will bring. Buat et al. followed this discussing the importance of wide surveys with AKARI, and emphasising the difficulties of establishing the link between star formation, and discussing SED fitting using a combination of AKARISDSS-GALAEX observations with the CIGALE code.

Continuing the observational studies, Kim et al. reported observations of the PAH $3.3 \mu \mathrm{m}$ feature, suggesting that it could be used as an effective tracer of star formation activity, whilst stressing that it was still unclear how AGN activity is connected to the staf formation activity for different galaxy classes. Takagi et al. showing the uniqueness of AKARI data in the 8$24 \mu \mathrm{m}$ region for observing the properties of the ISMs and estimating SF rates from equivalent widths of $\mathrm{PAH}$ features showing how this could be used to differentiate the differences in the environment in the indi- vidual galaxies. Makiya and Goon Lee together discussed the properties of large samples of galaxies from the 5.4 square degree AKARI NEP-Wide survey, which contained about 115,000 sources, and presented correlations with their individual properties, as well as in clusters and groups. Ko et al. presented a mid-infrared view of red sequence galaxies, focussing on the NEP supercluster, also from the AKARI NEP-Wide survey. This topic was further explored by Shim \& Toba, showing that AKARI (N3-S11) observations of the merging cluster Abell 2255 are a very effective at studying excess galaxies in clusters. Goto et al. showed that AGN evolution and star formation could be very well studied using the wide spectral range of AKARI, which provided significant advantages over data available from previous missions, particularly in the redshift range 0 $<\mathrm{z}<2.2$. Toba et al. then showed impressive midIR luminosity functions taken from the All Sky survey catalogue, which showed that most (all?) AGN's in the local Universe are dust enshrouded, and that the structure of the dust torus is related to the luminosity.

Moving to the high redshift Universe, Im et al. and Sedgwick et al. reported impressive observations that together have led to the detection of red-shifted $\mathrm{H} \alpha$ in $\sim 200$ objects to redshifts 6.4 (in a QSO), revealing the evolution of supermassive black holes in the redshift range out to $\mathrm{z} \sim 6.4$, but surprisingly showing little 
evidence for substantial growth at earlier epochs. Matsuoka et al. discussed the cosmic optical background, suggesting that a dominant component of it resulted from the integrated radiation of stellar nucleosynthesis of galaxies with redshifts $>10$, complementing work on the far-IR background by Matsuura et al. (2011).

\section{SYNERGIES AND THE FUTURE}

Blain et al. compared the data from the WISE and AKARI missions, showing the results from combining the two data sets to obtain wide spectral coverage. Pearson et al. showed results form ESA's Herschel satellite, and discussed how the synergy with AKARI would naturally lead on to the future SPICA mission. Nakagawa et al. presented an update on the present status of SPICA (Swinyard et al., 2009), which has a target launch window after 2020 should it be approved, and would be the natural successor to AKARI.

\section{9. 'JEJUISMS' OVERHEARD DURING THE CONFERENCE}

- DOGs are the future - Hideo Matsuhara

- For AGN the data are 'ratty', but overall they kind of make sense - George Helou

- Wow! - Takao Nakagawa

- I am sure that AKARI Deep Field South has a very bright future - Dave Clements

- Cosmologists and Theoreticians say it's all about AGN feedback - Ji Hoon Kim

- It is all in the fit parameter covariance matrix George Helou

- The green valley lies between the blue and the red clouds - Veronique Buat

- Buried AGNs are elusive - Masatoshi Imanishi

- Asteroids are not thermally differentiated, but contain a pristine record of the Solar System's formation - Fumihiko Usui

- PAHs are mostly in neutral state - Ho-Gyu Lee

- I am Steve Serjeant - Chris Pearson

- Cluster-scale merging suppresses star formation - Hyun Jin Shim
- You need to check that your model has good coverage of the parameters in your model - Denis Burgarella

- AKARI has opened an new era for galaxy cluster studies - Gwang Ho Lee

- Hideo Matsuhara in commenting to Glenn White after the latter's attempt at a multi-lingual English/Japanese/Korean presentation: 'I enjoyed your Japanese very much - but I needed to translate it back into English again to be able to understand you'

\section{ACKNOWLEDGEMENTS}

It is a pleasure to thank, on behalf of the participants, the Co-organisers, Hyung Mok Lee (SNU, Korea) and Hidehiro Kaneda (Nagoya, Japan); the Science Co-ordinating Committee (Andrew Blain (Leicester, UK, Denis Burgarella (LAM, France), George Helou (IPAC, USA, Myungshin Im (SNU, Korea), Hideo Matsuhara (ISAS, Japan), Margaret Meixner (STSci, USA), Thomas Mueller (MPE, Germany), Takao Nakagawa (ISAS, Japan); Devendra Ojha (TIFR, India), Takashi Onaka (Tokyo, Japan), Soojong Pak (Kyunghee University, Korea), Chris Pearson (RAL, UK), Glenn White (Open University and RAL, UK); the Local Organising Staff (Myungshin Im (SNU, Korea), Daisuke Ishihara (Nagoya, Japan), Woong-Seong Jeong (SNU, KASI, Korea), Hidehiro Kaneda (Nagoya, Japan), Hyoin Lee (SNU, Korea), Yoshiki Matsuoka (Nagoya, Japan), Hyunju Noh (SNU, Korea), Shinki Oyabu (Nagoya, Japan).), and the Sponsors The Center for Exploration of Origin of the Universe, Seoul National University; Astronomy Program, Seoul National University; Nagoya University Global COE Program: Quest for Fundamental Principles in the Universe; Division of Particle and Astrophysical Science, Nagoya University and The Institute of Space and Astronautical Science, Japan Aerospace Exploration Agency. The meeting was hosted by the Frontier Physics Research Division (SNU, Korea) and the institutions above.

\section{REFERENCES}

Doi, Y., Komugi, S., Ikeda, N., Kato, D., et al., 2012, in preparation

Ishihara, D., Onaka, T., Kataza, H., Salama, A., 2010, The AKARI/IRC Mid-Infrared All-Sky Survey, A\&A, 514, A1 
Murakami, H., Baba, H., Barthel, P. D., et al., 2007, The Infrared Astronomical Mission AKARI, PASJ, 59, S369

Matsuura, S., Shirahata, M., Kawada, M., Takeuchi, T., et al., 2011, Detection of the Cosmic Far-infrared Background in AKARI Deep Field South, ApJ, 737, 2

Negrello, M., Serjeant, S., Pearson, C., Takagi, T. et al., 2009, Photometric Redshift Accuracy in AKARI Deep Surveys, MNRAS, 394, 375

Swinyard, B., Nakagawa, T., Merken, P., Royer, P., et al., 2009, The Space Infrared Telescope for Cosmology and Astrophysics: SPICA a Joint Mission between JAXA and ESA, Experimental Astronomy, 23, 193

Wada, T., Matsuhara, H., Oyabu, S., \& Takagi, T., 2008, AKARI/IRC Deep Survey in the North Ecliptic Pole Region, PASJ, 60, S517

Ward-Thompson, D., Di Francesco, J., Hatchell, J., Hogerheijde, M. R., et al., 2007, The James Clerk Maxwell Telescope Legacy Survey of Nearby Starforming Regions in the Gould Belt, PASP, 119, 855

White, G. J., Pearson, C., Braun, R., Serjeant, S., et al., 2010, A Deep Survey of the AKARI North Ecliptic Pole Field. I. WSRT $20 \mathrm{~cm}$ Radio Survey Description, Observations and Data Reduction, A\&A, 517, A54

Yamamura, I., Makiuti, S., Ikeda, N., Fukuda, Y., et al., 2010, AKARI/FIS All-Sky Survey Point Source Catalogues (ISAS/JAXA, 2010), VizieR On-line Data Catalog, 2298, 0 